\title{
Matrix element effects in angle-resolved photoemission from Bi2212: Energy and polarization dependencies, final state spectrum, spectral signatures of specific transitions and related issues
}

\author{
M. Lindroos ${ }^{1,2}$, S. Sahrakorpi ${ }^{1,2}$ and A. Bansil ${ }^{2}$ \\ 1 Tampere University of Technology, P.O. Box 692, FIN-33101 Tampere, Finland \\ 2 Physics Department, Northeastern University, Boston, Massachusetts 02115
}

\begin{abstract}
We have carried out extensive simulations of the angle-resolved photoemission (ARPES) intensity in Bi2212 within the one-step and three-step type models using a first-principles band theory framework. The focus is on understanding the behavior of emissions from the antibonding and bonding bands arising from the $\mathrm{CuO}_{2}$ bilayers around the $\bar{M}(\pi, 0)$ symmetry point. The specific issues addressed include: Dependencies of the photointensity on the energy and polarization of the incident light; character of the initial and final states involved as well as the spectrum of the relevant final states; and, changes in the spectral intensity as a function of the perpendicular component, $k_{\perp}$, of the momentum of the photoelectron. Considerable insight into the nature of individual transitions is adduced by examining the momentum matrix element for bulk transitions within the solid and by further decomposing this matrix element into contributions from various atomic sites and angular momentum channels. These results indicate that, via remarkable interference effects, the ARPES matrix element can in particular cases help zoom in on the properties of electrons excited from specific sites and/or angular momentum channels even in a complex material.
\end{abstract}




\section{INTRODUCTION}

It is well recognized that the photoemission process involves an interplay between the bulk and surface phenomena as the excited electron must be ejected from the surface in order to reach the detector. The intensity measured in an ARPES (angle-resolved photoemission spectroscopy) experiment, therefore, fundamentally involves the properties of the relevant initial and final states in the presence of the surface. As a result, the ARPES intensity can differ greatly from the spectral density of the initial state involved in the excitation. An understanding of this difference - the so-called 'matrix element effect', is essential for a satisfactory interpretation of the ARPES spectra, and this is particularly so in complex materials such as the high-Tc's 11 3.

Bearing these considerations in mind, we have carried out extensive simulations of the ARPES spectra in $\mathrm{Bi}_{2} \mathrm{Sr}_{2} \mathrm{CaCu}_{2} \mathrm{O}_{8}$ (Bi2212, BISCO) within the first-principles band theory framework. The pristine phase with the body centered tetragonal lattice structure is assumed; the computationally more demanding case of the orthorhombic and modulated lattices is hoped to be taken up in the future. We focus on the excitation of states in the vicinity of the Fermi energy $\left(E_{F}\right)$ around the $\bar{M}(\pi, 0)$ symmetry point which have been the subject of intense scrutiny and controversy in the high-Tc literature. For example, the issue of bilayer splitting - expected to be relatively large within the conventional LDA-based band theory picture at $\bar{M}$ in BISCO, has been debated for some time, even though a consensus in favor of the existence of a bilayer splitting appears to be emerging in the recent months [4] [6]. The fundamental question has been whether or not strong correlations localize electrons in the $\mathrm{CuO}_{2}$ layers, yielding a 2D electron gas and a zero bilayer splitting with significant implications for the mechanism of superconductivity in the cuprates. [7] This study gives insight into these and related aspects of the ground state electronic structure by delineating the connection between the ARPES spectra and the character of the underlying initial and final states.

For this purpose, we have developed the one-step methodology [8] for modeling the photoemission process so that arbitrarily complex systems with many basis atoms can be treated [1,2, 9 11]. The previous one-step work has been limited to a maximum of two atoms per unit cell [12] which is not adequate for the cuprates; BISCO, for example, involves 30 atoms per conventional unit cell even for the pristine lattice. In the one-step approach, the interaction of light with the solid is treated as a single quantum mechanical event and the artifical distinctions between the processes of excitation, transport and ejection of the photoelectron invoked in the earlier three-step models 13 17 are not made. The one-step scheme is thus inherently more satisfactory than the three-step model since the surface is incorporated in the latter in an ad hoc manner. On the other hand, it often tends to be difficult to identify the behavior of individual transitions within the one-step methodology where the initial and final states must be damped, causing these states to broaden and overlap, blurring distinctions between various transitions. For this reason, we have also developed the three-step model for complex lattices [17]. Useful insight is adduced here into different contributions to the ARPES spectrum of BISCO by examining the momentum matrix element for bulk transitions in the solid in the spirit of the three-step approach.

The specifics of some of the main issues addressed in this article and highlights of the new results presented are as follows. The dependencies of the ARPES intensity on the energy and 
polarization of the incident light are clarified for the excitation of states near the $\bar{M}$ point. The response of the antibonding and bonding combinations of states from the $\mathrm{CuO}_{2}$ bilayers is found to display a strong energy and polarization dependence. Moreover, the behavior of the antibonding and bonding states differs significantly so that by a judicious choice of these experimental parameters even closely placed bilayer bands could be distinguished. Also, photon energies where the intrinsic cross-section for exciting certain states of the pristine lattice is large will be best suited for studying these states; in contrast, when this cross-section is relatively small, the effects of deviations from the tetragonal symmetry of the system via various distortions and modulations will become more prominent. Further, we show how some systematics of the polarization dependencies can be understood in the cuprates via straightforward arguments involving the symmetries of the initial and final states.

Turning to questions related to individual transitions, we discuss the nature of the momentum matrix element for bulk excitations within a three-step type model in terms of contributions from various basis atoms and different angular momentum channels. The character of the bonding and antibonding initial states as well as that of the relevant final states at $\bar{M}$ is clarified. An analysis of these results reveals dramatically how different contributions to the momentum matrix element can interfere constructively or destructively to enhance or supress the weights of photoelectrons excited from particular atomic sites and/or specific angular momentum channels. This remarkable result which, to our knowledge, has not been recognized previously in the literature, hints that the ARPES matrix element may allow us to zoom in on the properties of very specific transitions even in complex unit cell materials.

Concerning relevant literature, Refs. [1, 2, 9, 10, 18, 19] discuss some of our earlier work on BISCO, $\mathrm{Nd}_{2-x} \mathrm{Ce}_{x} \mathrm{CuO} \mathrm{O}_{4}$ (NCCO), $\mathrm{YBa}_{2} \mathrm{Cu}_{3} \mathrm{O}_{7}$ (YBCO) and $\mathrm{YBa}_{2} \mathrm{Cu}_{4} \mathrm{O}_{8}(\mathrm{Y} 124)$. In particular, Ref. [1] emphasizes the importance of matrix element effects in ARPES spectra, while Ref. [3] undertakes an extensive comparison between the one-step predictions over a wide region in the momentum space when the initial state energy is held fixed at the $E_{F}$ with the corresponding experimental ARPES results giving the Fermi surface map in BISCO. There is of course a large body of available work on the photoemission theory and Refs. [20 25] would provide a brief selection. On the experimental side as well the ARPES studies of BISCO are far too numerous to be cited with any completeness, and we refer only to a few representative papers. [4, 6, 26, 35]

An outline of this article as is follows. The introductory remarks are followed in Section II by a consideration of formal matters; a few equations for the one-step model are presented (Subsection IIA), and the problem of computing the momentum matrix element in a general lattice is formulated (Subsection IIB). Section III delineates the relevant features of the lattice geometry (Subsection IIIA) and the band structure of BISCO (Subsection IIIB). The results of Sections II and III serve as a source of frequent reference in the subsequent discussion. Section IV is divided into several subsections to go over various salient features of the ARPES spectra from $\bar{M}$. The aspects addressed concern: Photon energy dependence (Subsection IVA); Effect of final state width (Subsection IVB); Polarization dependence (Subsection IVC); and, the final state band structure and momentum matrix element (Subsection IVD). Section $\mathrm{V}$ takes up the question of the nature of the transition matrix element and the origin of specific spectral features in terms of the characters of the initial and final 
states and is divided into three parts dealing with: Contributions of different atomic sites (Subsection VA); contributions of various angular momentum channels (Subsection VB); and, comments on the $k_{\perp}$ dependence of the spectral intensities (Subsection VC). Finally, Section VI summarizes our results and makes a few concluding remarks.

\section{THEORETICAL CONSIDERATIONS}

\section{A. General aspects}

We begin by recalling that the ARPES intensity in the one-step model can be expressed as $[22,8$

$$
I=-\frac{1}{\pi} \operatorname{Im}<\mathbf{k}_{\|}\left|G_{2}^{+} \Delta G_{1}^{+} \Delta^{\dagger} G_{2}^{-}\right| \mathbf{k}_{\|}>
$$

where the ket $\mid \mathbf{k}_{\|}>$denotes a free electron state with momentum, $\mathbf{k}_{\|}$, parallel to the crystal surface, $G_{2}\left(G_{1}\right)$ is the retarded (+) or advanced (-) one-electron Green function for the final (initial) state and

$$
\Delta=e \hbar / 2 m c(\mathbf{p} \cdot \mathbf{A}+\mathbf{A} \cdot \mathbf{p})
$$

is the interaction Hamiltonian in terms of the vector potential $\mathbf{A}$ of the incident photon field and the electron momentum operator $\mathbf{p}$.

For our purposes, we rewrite Eq. [1 by using the representation

$$
-\frac{1}{\pi} \operatorname{Im} G_{1}^{+}=\sum_{i}\left|i>B_{i i}<i\right|
$$

of the Green function $G_{1}$ as a sum over the spectral functions $B_{i i}$ for various initial states $i$ which yields

$$
\begin{aligned}
I & =<\mathbf{k}_{\|}\left|G_{2}^{+} \Delta \sum_{i}\right| i>B_{i i}<i\left|\Delta^{\dagger} G_{2}^{-}\right| \mathbf{k}_{\|}> \\
& =\sum_{i} B_{i i}<\mathbf{k}_{\|}\left|G_{2}^{+} \Delta\right| i><i\left|\Delta^{\dagger} G_{2}^{-}\right| \mathbf{k}_{\|}> \\
& =\sum_{i} B_{i i}|<f| \Delta|i>|^{2}
\end{aligned}
$$

Here we have replaced $G_{2}^{-} \mid \mathbf{k}_{\|}>$by the final state wavefunction $\mid f>$. Substituting form for $\Delta$ in Eq. 2, and neglecting the term proportional to $\nabla \cdot \mathbf{A}$ by assuming that the vector potential A varies smoothly, i.e. wavelength of light is much larger than the unit cell dimensions [36], we obtain

$$
I=e \hbar / m c \sum_{i} B_{i i}|\mathbf{A} \cdot<f| \mathbf{p}|i>|^{2}
$$

Notably, the summation over $i$ not only encompasses different initial state bands, but also involves an integration over $\mathbf{k}_{\perp}$. A computationally practical formula follows by invoking the identity 


$$
[H, p] \equiv H p-p H=-i \hbar \nabla V
$$

which is valid when the potential term is $\mathbf{p}$ independent. The matrix element in Eq. 7 is easily recast using Eq. 8 as

$$
\mathbf{A} \cdot<f|\mathbf{p}| i>=\frac{-i \hbar}{E_{i}-E_{f}} \mathbf{A} \cdot<f|\nabla V| i>
$$

where $E_{i}$ and $E_{f}$ are the initial and final state energies, respectively.

\section{B. Momentum matrix element}

The nature of the photointensity based on the one-step Eq. 1 can be delineated in terms of the momentum matrix element $\langle f|\mathbf{p}| i>$ of Eq. [1. However, the wavefunctions $| i>$ and $\mid f>$ occuring in Eqs. 3 - 9 are complicated quantities which include multiple scattering effects in the presence of the surface and damped initial and final state propagators. Although the full one-step calculation should always be kept in mind, insight into the results may be obtained by simplifying the situation and replacing $\mid i>$ and $\mid f>$ by the corresponding Bloch wavefunctions $\tilde{\psi}$ in the bulk crystal and the resulting matrix element $<\tilde{\psi}_{f}|\mathbf{p}| \tilde{\psi}_{i}>$ for optical transitions. It should be emphasized that the optical matrix element must in general be supplemented with other information specific to the photoemission experiment (e.g. symmetry of the final state) before connection with a particular measurement can be made. This issue will be addressed as needed in the remainder of this article.

The formalism for evaluating the optical matrix element for a general lattice within the KKR scheme is given in Refs. [17,37]; an outline of aspects relevant for the purposes of this article is as follows. The starting point is the KKR wavefunction for a general lattice which can be expressed within the unit cell as [38,39]

$$
\tilde{\psi}(\mathbf{r})=\sum_{L, \beta} i^{l} C_{L}^{\beta} R_{l}^{\beta}(r) Y_{L}(\Omega)
$$

where $L \equiv(l, m)$ is a composite angular momentum index and $\beta$ denotes different basis sites.

$C_{L}^{\beta}$ are expansion coefficients and $R_{l}^{\beta}(r)$ is the radial part of the Bloch wavefunction on site $\beta$ in angular momentum channel $L . Y_{L}(\Omega)$ are real spherical harmonics. Using expressions of Eq. 10 for the initial and final states yields

$$
<\tilde{\psi}_{f}|\mathbf{p}| \tilde{\psi}_{i}>=\sum_{\alpha, \beta} \sum_{L, L^{\prime}} \hat{\mathbf{e}}_{\alpha} i^{l-l^{\prime}-1} C_{L^{\prime}}^{{ }^{*}} C_{L}^{\beta} B_{l, l^{\prime}}^{\beta} \mathcal{G}_{L, L^{\prime}}^{\alpha}
$$

where the primed indices refer to the final state and $\hat{\mathbf{e}}_{\alpha}$ is a unit vector along the direction $\alpha . B_{l, l^{\prime}}^{\beta}$ involves an integral over the radial part of the initial and final state wavefunction and their derivatives and is given by 


$$
B_{l, l^{\prime}}^{(\beta)}=\left\{\begin{array}{cc}
\int_{0}^{r_{\beta}}( & \left.P_{l^{\prime}}^{\star} P_{l}^{\prime}-\frac{l+1}{r} P_{l^{\prime}}^{\star} P_{l}\right) d r \\
& +\frac{1}{E_{i}-E_{f}} \\
& \times\left\{P_{l^{\prime}}^{\star \star} P_{l}\left[\frac{(l+1)^{2}}{r^{2}}-E_{i}\right]\right. \\
& +\frac{l+1}{r}\left[P_{l^{\prime}}^{\prime \star} P_{l}-P_{l^{\prime}}^{\star} P_{l}^{\prime}\right] \\
& \left.-P_{l^{\prime}}^{\prime \star} P_{l}^{\prime}\right\}_{r=r_{\beta}} \\
\int_{0}^{r_{\beta}} & \left(P_{l^{\prime}}^{\star \star} P_{l}^{\prime}+\frac{l}{r} P_{l^{\prime}}^{\star} P_{l}\right) d r \\
& +\frac{1}{E_{i}-E_{f}} \quad l^{\prime}=l+1 \\
& \times\left\{P_{l^{\prime}}^{\star} P_{l}\left[\frac{l^{2}}{r^{2}}-E_{i}\right]\right. \\
& +\frac{l}{r}\left[P_{l^{\prime}}^{\prime \star} P_{l}-P_{l^{\prime}}^{\star} P_{l}^{\prime}\right] \\
& \left.-P_{l^{\prime}}^{\prime \star} P_{l}^{\prime}\right\}_{r=r_{\beta}}
\end{array}\right.
$$

Here, $P_{l}(r) \equiv r R_{l}^{\beta}(r), r_{\beta}$ is the radius of the $\beta^{t h}$ muffin-tin sphere, $E_{i}$ and $E_{f}$ have been defined in connection with Eq. 9 above, and

$$
\mathcal{G}_{L, L^{\prime}}^{\alpha}=\left\{\begin{array}{ll}
\sqrt{\frac{4 \pi}{3}} \int Y_{L^{\prime}}(\Omega) Y_{1, \alpha}(\Omega) Y_{L}(\Omega) d \Omega & l^{\prime}=l \pm 1 \\
0 & l^{\prime} \neq l \pm 1
\end{array},\right.
$$

$\mathcal{G}_{L, L^{\prime}}^{\alpha}$ are seen to contain angular momentum selection rules for dipole transitions. $Y_{1, \alpha}$ for $\alpha$ values of $-1,1$ and 0 is proportional to $x, y$ and $z$, respectively.

It is convenient to decompose the sum over $\beta$ on the right hand side of Eq. 11 for the $\alpha^{\text {th }}$ component as

$$
\begin{aligned}
M_{\alpha} \equiv<\tilde{\psi}_{f}\left|p_{\alpha}\right| \tilde{\psi}_{i}> & =\sum_{\gamma} M_{\alpha}^{\gamma} \\
& =\sum_{\gamma} \sum_{\delta} M_{\alpha}^{\gamma, \delta} \\
& =\sum_{\gamma} \sum_{\delta} \sum_{L, L^{\prime}} M_{\alpha, L, L^{\prime}}^{\gamma, \delta}
\end{aligned}
$$

with

$$
M_{\alpha, L, L^{\prime}}^{\gamma, \delta}=i^{l-l^{\prime}-1}\left(C_{L^{\prime}}^{\gamma, \delta}\right)^{*} C_{L}^{\gamma, \delta} B_{l, l^{\prime}}^{\gamma, \delta} \mathcal{G}_{L, L^{\prime}}^{\alpha}
$$

The indices $\gamma$ and $\delta$ in Eqs. 14 16 are keyed in with the pristine (tetragonal) crystal structure of BISCO detailed in Section IIIA below, and taken together, encompass the sum over all atoms $\beta$ in the unit cell in Eq. 10. After the sum over the angular momentum channels $L$ and $L^{\prime}$ in Eq. 16 has been carried out, the index $\delta$ which only takes two values (1 and 2) in Eq. 15 sums over the contributions of pairs of atoms (other than the $C a$ atom) related by mirror symmetry of the lattice with respect to the $C a$ layer. In this sense, $\delta$ may be 
thought of as a "pairing" index. $\gamma$ then takes on eight distinct values which include seven pairs of atoms $\left(\mathrm{Bi}, \mathrm{O}_{\mathrm{Bi}}, \mathrm{Sr}, \mathrm{O}_{\mathrm{Sr}}, \mathrm{Cu}, \mathrm{O}_{\mathrm{Cu}, \mathrm{x}}, \mathrm{O}_{\mathrm{Cu}, \mathrm{y}}\right)$ and the $\mathrm{Ca}$ atom as a "site index". The aforementioned indexing is by no means unique and other useful variations can be envisioned in the present case and certainly for other lattice types.

\section{RELEVANT FEATURES OF THE LATTICE GEOMETRY AND ELECTRONIC STRUCTURE OF BISCO}

\section{A. Lattice structure}

Since various structural features of the lattice are invoked frequently in the discussion, it is appropriate that we state our nomenclature clearly. For this purpose, two different cross-sections through the conventional body centered tetragonal unit cell, together with the detailed arrangement of atoms in different layers, are shown in Fig. 1. The distinct atom "pairs" to go with the index $\gamma$ in Eqs. 14-16 are identified in Fig. 1(c). Values of $\gamma$ from 2-8 involve an atom and its mirror partner reflected in the $C a$ layer, yielding a total of 15 basis atoms per primitive lattice point or 30 atoms in the conventional unit cell. The $\mathrm{Ca}$ atom $(\gamma=1)$ is seen to lie at the center of symmetry in the upper part of Fig. 1(a). The two $\mathrm{CuO}_{2}$ planes sit above and below the $\mathrm{Ca}$ layer and consist of the two $\mathrm{Cu}$ atoms in the basis set denoted by the $(\gamma, \delta)$ indices $(2,1)$ and $(2,2)$. $O_{C u, x}$ and $O_{C u, y}$ are given similarly by $\gamma=3$ and 4 , each with $\delta=1,2$; note that although $O_{C u, x}$ and $O_{C u, y}$ are identical in terms of the crystal potential in the tetragonal case, it is still useful to make this distinction because states along $x$ and $y$ will in general respond differently depending upon the direction of polarization of the incident light. Next come the $\operatorname{SrO}$ layers with $\operatorname{Sr}(\gamma=5)$ and the associated apical $O_{S r}$ atoms $(\gamma=6)$, followed by the $B i O$ layers involving the $B i(\gamma=7)$ and $O_{B i}(\gamma=8)$ atoms.

\section{B. Electronic structure}

Fig. 2 shows the energy bands in BISCO along the symmetry lines $\Gamma-M-X-\Gamma$. The crystal potential underlying these computations differs somewhat from the selfconsistent LDA potential in that the $\mathrm{Bi}-\mathrm{O}$ pockets around the M-point have been lifted above the $E_{F}$ in order to account for their absence in the ARPES measurements. The two bands in the vicinity of the $E_{F}$ are related to the $C u O_{2}$ planes; the splitting between $a$ and $b$ is the bilayer splitting arising from interaction between the two $\mathrm{CuO}_{2}$ planes. At the M-point, the wavefunction of the upper band $a$ is antibonding or antisymmetric with respect to the $\mathrm{Ca}$ plane (along the $z$ direction) while that of the lower band $b$ is bonding or symmetric. The size of the bilayer splitting is generally larger along $\Gamma-M$ compared to the $\Gamma-X$ direction. We have chosen to work in the present simulations with a potential that yields a rather large bilayer splitting because this makes it easier to identify the signatures of the bonding and antibonding bands $b$ and $a$ in the theoretical ARPES spectra [40]. However, computations have been repeated for numerous other cases where the bilayer splitting was

varied by placing suitable potential barriers between the $\mathrm{CuO}_{2}$ layers, and the results indicate 
that our conclusions concerning the nature of these states and their behavior in the ARPES spectra are robust to such variations.

The analysis of the recent high resolution ARPES spectra leaves little doubt that the Fermi surface of BISCO consists of two distinct sheets [1 3]. One is the standard large hole-like sheet centered around the $X$ or $Y$ symmetry point, and a second sheet arising from a band lying very close the the $E_{F}$ which could be either slightly hole- or electron-like. The precise position of the $E_{F}$ will depend of course on the doping level of the system. In any event, it is often useful to avoid convoluting the computed spectra with the Fermi function so that the nature of the spectra over a wider range of $\mathbf{k}_{\|}$and energy values, including unoccupied states above the $E_{F}$, can be elucidated. Notably, the $\mathbf{k}_{\perp}$ dispersion of bands terminating at points $a$ and $b$, even though it varies with $\mathbf{k}_{\|}$, is quite small (order of a few meV at $\bar{M}$ ) and is not important in much of our discussion.

\section{SALIENT FEATURES OF THE ARPES SPECTRA FROM THE $\bar{M}$ POINT}

\section{A. Photon energy dependence of emission intensity from bonding and antibonding states}

Fig. 3 shows the ARPES intensity from the antibonding and bonding states $(a$ and $b)$ at the $\bar{M}$ point as a function of the photon energy. For our illustrative purposes we have taken the light to be polarized such that only the $x$-component of the vector potential $\mathbf{A}$ is non-zero. The initial state width corresponds to $\Sigma_{i}^{\prime \prime}=100 \mathrm{meV}$ [1]. The final state width is set using $\Sigma_{f}^{\prime \prime}=1 \mathrm{eV}$ - we return to this point in Section IVB below. The emission intensity is seen to change dramatically with photon energy. The bonding state emits strongly around 18 and $23 \mathrm{eV}$ with a feature around $33 \mathrm{eV}$, while the antibonding state possesses a somewhat broad region of high emissions extending from 14-26 eV. The intensity for both states is relatively small from 27-40 eV and below $11 \mathrm{eV}$. These results have significant implications for ARPES experiments. For example, emission maps from the $E_{F}$ can be expected to look quite different at various photon energies; at an energy such as $22 \mathrm{eV}$, the emissions from the pristine lattice will be dominant, while at an energy such as $28 \mathrm{eV}$, the contributions due to modulations of the underlying symmetry (e.g. orthorhombic distortion, superlattice modulation, etc.) will generally become more prominent [3]. Energies such as 18 or 23 $\mathrm{eV}$ will tend to highlight the bonding state while the antibonding state will likely be more intense around $22 \mathrm{eV}$, effects of final state broadening and other factors notwithstanding. We should particularly keep in mind that there are inherent uncertainities of a few eV's in locating the final states in the first principles computations, and that the positions of various features in Fig. 3 will generally possess similar uncertainty. We have carried out additional computations (not shown in the interest of brevity) and find that the aforementioned type of variations in intensity which selectively highlight different aspects of the electronic structure continue to be manifest at photon energies much higher than the $40 \mathrm{eV}$ upper limit in Fig. 3 . 


\section{B. Effect of final state width}

Fig. 4 shows how the plot of Fig. 3 for the antibonding initial state changes when the final state width varies corresponding to $\Sigma_{f}^{\prime \prime}$ values from $20 \mathrm{meV}$ to $2 \mathrm{eV}$ [41]. The results for small $\Sigma_{f}^{\prime \prime}$ make it clear that the spectrum is intrinsically made up of numerous transitions with different weights. With increasing $\Sigma_{f}^{\prime \prime}$ these transitions broaden and overlap to produce varying spectral shapes of Fig. 4. For $\Sigma_{f}^{\prime \prime}<100 \mathrm{meV}$, little change in shape occurs because the spectra are controlled by the initial and not the final state width, the former having a constant value given by $\Sigma_{i}^{\prime \prime}=100 \mathrm{meV}$ in all cases. Note that to reproduce the observed linewidths, $\Sigma_{f}^{\prime \prime}$ used in the computations will need to account not only for the damping of the final state reflected via the imaginary part of the self-energy, but also the effects of experimental parameters such as the temperature of the measurement and the acceptor angle of the analyzer (typically of order of a few tenths of a degree in the high resolution ARPES setups). In this sense, $\Sigma_{f}^{\prime \prime}$ is perhaps better viewed as a computational rather than a physical parameter in the theory with values ranging from $1-4 \mathrm{eV}$, where $4 \mathrm{eV}$ is appropriate for 40-60 eV photons 42,43. Incidentally, computations become more demanding as the value of $\Sigma_{f}^{\prime \prime}$ decreases because the mean free path of the outgoing electron increases and an exponentially larger number of layers must be included in the calculations to obtain a converged result.

\section{Polarization dependence}

Insofar as the polarization dependence is concerned, note first that in Fig. 5 light is assumed incident normally to the surface and that the detector lies in the $x-z$ plane with the $x$-axis defined by the $\mathrm{Cu}-\mathrm{O}_{x}$ bond direction; the polarization vector of light is varied holding all other experimental parameters fixed. The inserts in the shape of number eight are polar plots of the intensities of various peaks $\left(a, b, c\right.$ and $\left.c^{\prime}\right)$ as a function of the azimuthal angle $\phi$ between the polarization vector and the $x$-axis. The intensity is maximum for all peaks at $\phi=0$, i.e. when the light is polarized along the $C u-O_{x}$ bonds. Under a $90^{\circ}$ rotation the intensity nearly vanishes as the polarization vector turns perpendicular to the plane of the detector.

Insight into the polarization dependence discussed in the preceding paragraph can be gained by examining the behavior of Eq. 7 for the geometrical setup of Fig. 5. Since the detector lies in the $x-z$ plane of mirror symmetry, the final state $\mid f>$ must obviously possess even symmetry with respect to this plane in order to be observable. On the other hand, $p_{x}$ or $p_{z}$ (or, equivalently, $\partial V / \partial x$ or $\partial V / \partial y$ from Eq. 9) is even but $p_{y}$ is odd under reflections in this mirror plane. It follows then that only the matrix elements $\left\langle f\left|p_{x}\right| i\right\rangle$ and $<f\left|p_{z}\right| i>$ can be non-zero for even initial states while for odd initial states only $<f\left|p_{y}\right| i>$ can be non-zero. We now express $\mathrm{A}$ in spherical polar coordinates as

$$
\mathbf{A}=A_{0} \hat{\mathbf{e}}=A_{0}\left(\cos \phi \cos \theta \hat{\mathbf{e}}_{x}+\sin \phi \cos \theta \hat{\mathbf{e}}_{y}+\sin \theta \hat{\mathbf{e}}_{z}\right)
$$

where $A_{0}$ is the amplitude, $\theta$ and $\phi$ are the polar and azimuthal angles of the incoming light beam, and $\hat{\mathbf{e}}_{\alpha}$ are unit vectors along the Cartesian axis. For the setup of Fig. 5, $\theta=0$, and Eq. 0 reduces to 


$$
I=e \hbar / m c \sum_{i} B_{i i}\left|A_{0}\left(\cos \phi \hat{\mathbf{e}}_{x}+\sin \phi \hat{\mathbf{e}}_{y}\right) \cdot<\psi_{f}\right|\left(\mathbf{p}_{x}+\mathbf{p}_{y}\right)\left|\psi_{i}>\right|^{2}
$$

It turns out that the antibonding state $a$ as well as the bonding state $b$ is even and, therefore, recalling the aforementioned symmetry arguments, only the $p_{x}$ term contributes and Eq. 19 yields

$$
I=e \hbar / m c \sum_{i} B_{i i}\left|A_{0}<\psi_{f}\right| p_{x}\left|\psi_{i}>\right|^{2} \cos ^{2} \phi
$$

which explains the shape of polar plots of Fig. 5 for states $a$ and $b$; states $c$ and $c^{\prime}$ must also be even in view of the shapes of the related polar plots. The preceding analysis is generalized straightforwardly to consider vector potentials with other non-zero components. If a nonzero $p_{z}$ component is included, Eq. 20 is modified with a radially symmetric contribution. In this vein, if the mirror symmetry plane is rotated by $45^{\circ}$ as is the case in YBCO (neglecting the effect of $\mathrm{Cu}-\mathrm{O}$ chains), that leads to polar plots which are rotated similarly. [18,44

Fig. 5 also shows the energy distribution curve (EDC) for $22 \mathrm{eV}$ photons when $\mathbf{k}_{\|}$is held fixed at $\bar{M}$. The EDC for the case when the initial state width is taken to be a small constant is given by the thin line for reference in order to reveal the spectral peaks clearly. More realistically, the initial state width increases with increasing binding energy. This effect is modelled by the EDC of the thick line which shows that much of the structure for binding energies greater than $\sim 100 \mathrm{meV}$ is washed out by lifetime effects.

\section{Final state band structure, momentum matrix element}

Fig. 6 compares the one-step ARPES intensity at the $\bar{M}$ point for the antibonding state $a$ with the corresponding result based on the bulk momentum matrix element of Eq. 14 and helps address a number of related issues. The small final state width used in these simulations allows the intrinsic spectral structure to be seen clearly. The final state bands shown as a function of $k_{\perp}$ in the lower portion of the figure provide a map of all available transitions since $k_{\perp}$ is not conserved in the photoemission process. [Note that the horizontal scale has been converted to refer to the photon energy.] It is striking that although these bands appear to form a semi-continuum, there are gaps in the underlying final state spectrum and the situation thus is quite unlike the free electron case 455. Also, $k_{\perp}$ dispersion is not always negligible with some bands dispersing as much as $0.5 \mathrm{eV}$ despite the small size of the $\mathrm{BZ}$ in the z-direction.

As already noted, it tends to be difficult to associate peaks in the one-step spectra with transitions to specific final states. The problem becomes exacerbated when states are broadened to incorporate finite lifetime effects. Considerable insight into the behavior of the ARPES matrix element can nevertheless be gained via the momentum matrix element

$<\tilde{\psi}_{f}|\mathbf{p}| \tilde{\psi}_{i}>$ of Eq. 11 for bulk transitions. We emphasize however that $\left\langle\tilde{\psi}_{f}|\mathbf{p}| \tilde{\psi}_{i}>\right.$ does not properly account for the processes of transport and ejection of the photoelectron as is the case in the one-step ARPES computation. Moreover, since $<\tilde{\psi}_{f}|\mathbf{p}| \tilde{\psi}_{i}>$ involves the bulk wavefuctions $\tilde{\psi}_{i}$ and $\tilde{\psi}_{f}$, modifications of these wavefunctions in the vicinity of the surface and the effect of possible formation of surface states are not incorporated. Even so, Fig. 6 shows that the one-step spectrum for the antibonding state $a$ is rather similar to that based 
on the momentum matrix element. Some differences in the intensities of certain peaks in the figure may be understood as consequences of the fact that the momentum matrix element has been evaluated at $k_{\perp}=0$ while in the one-step result $k_{\perp}$ value is in general non-zero and varies from $k_{\perp}=0$ to the zone boundaries at $k_{\perp}= \pm \pi / c$ for various peaks. Results for the bonding state $b$ (not shown for brevity) are similar in that the level of agreement between the one-step and the momentum matrix element based spectra is comparable to that of Fig. 6.

\section{NATURE OF TRANSITION MATRIX ELEMENTS AND ORIGIN OF SPECTRAL FEATURES IN TERMS OF CHARACTER OF INITIAL AND FINAL STATES}

\section{A. Contributions of different atomic sites and related interferences}

Contributions $M^{\gamma, \delta}$ of different atomic sites in the unit cell can be delineated via the decomposition of Eqs. 14 16. Table 1 and Fig. 7 provide illustrative examples of such an analysis. Considering $x$-polarized light, values of $M_{x}^{\gamma, \delta}$ for each of the 15 basis atoms for one particular transition from the states $a$ and $b$ at $\mathrm{M}$ are listed in Table 1. Fig. 7 on the other hand gives various significant contributions to all transitions from state $a$ over the energy range of $10-27 \mathrm{eV}$.

The strong influence of the mirror symmetry around the $C a$ layers is evident in Table 1. For the particular transition considered, the magnitudes of $M_{x}^{\gamma, \delta}$ are the same for the two mirror partners corresponding to the two $\delta$ values, but the phases differ by $\pi$. In fact, contributions cancel pairwise for the bonding state $b$, but interfere constructively for the antibonding state $a$. For many other transitions from $a$ and $b$ the situation is quite the opposite in that this sort of interference is destructive for $a$, but constructive for $b$. As we move away from $\mathrm{M}$, these cancellation effects become less perfect as the phases do not always differ exactly by $\pi$ and/or the magnitudes of the two mirror partners are no longer the same.

Figure 7 provides a somewhat broader perspective. Total matrix element $\left|M_{x}\right|$ obtained by summing over $\gamma$ and $\delta$ is depicted pictorially for many transitions from $a$ together with contributions from pairs of $\mathrm{Cu}, \mathrm{O}_{\mathrm{Cu}, x}, \mathrm{O}_{\mathrm{Bi}}$ and $\mathrm{O}_{S r}$ atoms; $M_{x}^{\gamma}$ for $\mathrm{Ca}, \mathrm{Sr}, \mathrm{Bi}$ and $\mathrm{O}_{\mathrm{Cu}, y}$ are less than 0.01 in magnitude and are not shown. Of a total of 64 possible transitions from state $a$ over the energy range of Fig. 7, only 20 transitions that are shown possess a significant intensity. A few of the transitions are identified by numbers 1-7 for convenient reference. As already noted above in connection with Table 1 , transition 1 is quite intense and arises almost completely from $O_{C u, x}$. Transition 4 contains an admixture of $O_{C u, x}$ and $\mathrm{Cu}$, while transition 3 contains additionally a contribution from $O_{B i}$. Transition 5 contains contribution from all these atoms as well as $O_{S r}$, but as indicated by downarrows, $\mathrm{Cu}$ and $O_{B i}$ give negative contributions yielding a net reduction in the total intensity. These examples would make it clear that constructive or destructive interference can take place not only for a given atomic pair, but also between different atomic sites. We emphasize that the intensity variations in Fig. 7 reflect changes in the character of the final states since the initial state for all transitions is fixed to be the same. 


\section{B. Contributions of various angular momentum channels and interference effects therein}

Further insight can be gained by considering the angular momentum dependence of the matrix element, i.e. the quantities $M_{x, L, L^{\prime}}^{\gamma \delta}$ of Eq. 17. For illustrative purposes, we consider first the aforementioned transition 1 with the help of Tables II-V, followed by a few comments concerning the behavior of transitions more generally via Fig. 8.

The weights in different angular momentum channels (defined as $\sum_{\delta}\left|C_{L}^{\gamma \delta}\right|^{2}$ ) for the initial states $a$ and $b$ associated with various atomic sites are given in Tables II and III, while the final state for transition 1 is characterized similarly in Table IV [46]. We emphasize that these weights depend upon details of how space within the unit cell is ascribed to different atoms and the size and shape of the interstitial region. For this reason, we should keep in mind that the results of Tables II-IV possess inherent uncertainty, even though these are representative of the character of the states involved. As expected, wavefunctions of both $a$ and $b$ are seen from Tables II and III to be predominantly $C u\left(d_{x^{2}-y^{2}}\right)$ and $O_{C u, x}\left(p_{x}\right)$ like; $O_{B i}$ as well as $O_{S r}$ states hybridize more strongly with the bonding level $b$ compared to $a$. The final state in Table IV continues to be weighted in favor of $C u$ and $O_{C u, x}$, but being 17.7 $\mathrm{eV}$ above the initial states, there is substantial change with respect to distribution among angular momentum channels and atomic sites.

We turn next to Table $\mathrm{V}$ and the matrix element for transition 1 from state $a$. Of the large number of possible terms $(\sim 300)$ under the summations in Eq. 16, only the few elements $M_{x, L, L^{\prime}}^{\gamma}$ listed in Table $\mathrm{V}$ possess substantial magnitude. The remaining elements are either zero due to selection rules or are negligibly small. The $p_{x} \rightarrow d_{x^{2}-y^{2}}$ channel on $O_{C u, x}$ is seen to be the strongest with a contribution (in arbitrary units) of 0.27 . It is most striking that the net contribution from $C u$ is only 0.02 even though the initial state is dominated by $C u$. This is the result of strong interference effects: On $C u$, different angular momentum channels interfere destructively as manifest in the positive and negative signs of elements, while on $O_{C u, x}$, there is a constructive interference yielding a large total intensity of 0.43. These observations hint that the ARPES matrix element can display remarkable site selectivity properties. For transition 1 under discussion, for example, almost all the photoelectrons emanate from $O_{C u, x}$ sites with little contribution from other sites in the crystal due to complicated interferences.

Fig. 8 considers all transitions from $a$ over the energy range of $10-27 \mathrm{eV}$. The total matrix element $\left|M_{x}\right|$ and the portion $\left|M_{x}^{\gamma}\right|$ associated with $O_{C u, x}$ in the top two panels of the figure are reproduced from Fig. 7 for ease of discussion; the total $O_{C u, x}$ intensity is decomposed into angular momentum channels in the three lower panels. By comparing the lengths of arrows in Figs. 8(a) and 8(b), we see that for most transitions (but not all) $O_{C u, x}$ component is strong. The effect however is not as dominant as in the case of transition 1; for example, only about 60 percent of transitions 2-4 come from $O_{C u, x}$ and transition 6 has essentially a zero $O_{C u, x}$ part. Moreover, even though different angular momentum channels interfere constructively for most transitions in that arrows are mostly pointed up in Figs. $8(\mathrm{c})-(\mathrm{e})$, this is not always the case as some arrows are pointed downwards. In particular, for transition 5 roughly half of the intensity in $p_{x} \rightarrow s$ channel is destroyed by inteference from $p_{x} \rightarrow d_{x^{2}-y^{2}}$ channel, and for transition 7 , these two channels destroy each other nearly completely. We note finally that, of the many factors involved in defining $M_{\alpha, L, L^{\prime}}^{\gamma \delta}$ on the 
right side of Eq. 17, much of the energy dependence depicted in Fig. 8 originates from the behavior of the wavefunction coefficients of the final states $\left(C_{L^{\prime}}^{\gamma \delta}\right)$; the Gaunt coefficients $\mathcal{G}_{L, L^{\prime}}^{\alpha}$ are constants and the quantities $B_{l, l^{\prime}}^{\gamma, \delta}$ involving integrals of radial wavefunctions are slowly varying functions of energy.

\section{C. $k_{\perp}$ dependence}

So far we have focused on the excitation of states $a$ and $b$ at the $\mathrm{M}(\pi, 0,0)$ point, i.e. for $k_{\perp}=0$. Changes in the momentum matrix element as a function of $k_{\perp}$ for several selected transitions from the initial state $a$ are considered in Fig. 9. Transitions 1-4 have been discussed previously in connection with Figs. 7 and 8. Transitions $2 \mathrm{~b}$ and 3b however, which lie quite close in energy to transitions 2 and 3, respectively, were not shown before since their intensity is zero or near-zero at $k_{\perp}=0$, but as Fig. 9 shows, this is not the case for $k_{\perp} \neq 0$. Transitions 1 and 4 decrease slightly in intensity as $k_{\perp}$ increases from zero to $\pi / c$. On the other hand, transitions 2, 2b, 3 or $3 \mathrm{~b}$ undergo large changes. Observable effects after spectra are smoothed with realistic broadenings of the order of a few eV's will likely be much smaller since the intensities of transitions 2 and $2 \mathrm{~b}$ (or similarly 3 and $3 \mathrm{~b}$ ) taken together are expected to change little with $k_{\perp}$.

We have carried out additional computations at $\mathbf{k}$-points on the $\bar{\Gamma}-\bar{M}$ line away from $\bar{M}$. The results continue to display complicated interference effects involving pairs of atoms and/or various atomic sites, essentially along the lines of the preceding commentary concerning emissions from $\bar{M}$, and require little further elaboration.

\section{SUMMARY AND CONCLUSIONS}

We have investigated the nature of the ARPES spectrum from BISCO using the firstprinciples band theory framework. Our focus is on understanding emission from electronic states along the $\bar{\Gamma}-\bar{M}$ symmetry line and particularly from the bonding and antibonding combinations of bands formed from the states associated with the $\mathrm{CuO}_{2}$ bilayers which have been the subject of considerable recent discussion and controversy. Salient features of the ARPES spectrum delineated are as follows.

1. The intensity of the antibonding as well as the bonding state at $\bar{M}$ displays a strong dependence on photon energy even though the two states differ significantly from each other in this respect. This result indicates that the bonding state will be highlighted at certain photon energies while the antibonding state will be intense at other energies; moreover, at photon energies where emission from either state is relatively weak, we would expect the effects of deviations of the underlying lattice from perfect tetragonal symmetry (i.e. via various modulations) to become more visible.

2. The spectra depend strongly on the polarization of the incident light. At the $\bar{M}$ point, for states in the vicinity of the $E_{F}$, the intensity is maximum when light is polarized along the $x$ - or the $C u-O_{x}$ bond direction, but becomes nearly zero upon a $90^{\circ}$ rotation of the polarization vector perpendicular to the plane of the detector. Simple arguments are presented to explain the $\cos ^{2} \phi$ (where $\phi$ is the angle between the $x$-axis and the polarization 
vector) variation observed in the one-step computations which yield a polar intensity plot in the shape of the number eight in BISCO and other cuprates.

3. An examination of the final states indicates that, despite the appearance of a semicontinuum, there are gaps in the final state spectrum and that the $k_{\perp}$ dispersion of some bands is as large as $0.5 \mathrm{eV}$ even though the Brillouin zone size is small along the z-direction. The situation thus is quite unlike the free electron case.

4. We show how considerable insight into the nature of the specific ARPES excitations can be adduced in terms of the momentum matrix element for bulk transitions within the solid in combination with constraints appropriate for a particular ARPES measurement. Such transition specific information tends to be difficult to ascertain within the more comprehensive one-step framework, especially in the presence of finite initial and final state dampings.

5. With the preceding point in mind, we have studied the nature of the momentum matrix element for emission from the $\mathrm{CuO}_{2}$ plane related antibonding state $a$ and the bonding state $b$ at $\bar{M}$ at length over the transition energy range of 10-27 eV. We show how the

matrix element, $|M|^{2} \equiv\left|<\tilde{\psi}_{i}\right| \mathbf{p}\left|\tilde{\psi}_{f}>\right|^{2}$, can be decomposed usefully into contributions from various atomic "pairs" in the unit cell (i.e. an atom and its mirror partner obtained by reflection through the $\mathrm{Ca}$ layer about which the BISCO lattice is symmetric), and further into different angular momentum channels. Contributions from the two atoms in the aforementioned atomic pairs display remarkable effects of interfering constructively for some transitions and destructively for others. The net contribution from the $O_{C u, x}$ pairs seems to dominate the spectrum in many cases even though the initial and final states involved possess large weights on the $\mathrm{Cu}$ sites. These strong interference effects continue to be present in different angular momentum channels. The analysis indicates that many transitions are controlled essentially by just the single $p_{x} \rightarrow d_{x^{2}-y^{2}}$ channel on the $O_{C u, x}$ sites. This striking result hints that the ARPES matrix element may possess most interesting site selectivity properties.

6. The intensity of many transitions increases (or decreases) substantially as a function of $k_{\perp}$. However, these variations are usually coupled with nearby transitions whose intensity decreases (or increases) so that the net contribution is generally not expected to show a strong $k_{\perp}$ dependence.

In summary, the present analysis makes it clear that the complicated nature of the ARPES matrix element in complex materials hides considerable potential for extracting useful information via this spectroscopy about the nature of specific states. Studies of other cuprates along these lines should allow an identification of robust aspects of the electronic structure related to the $\mathrm{CuO}_{2}$ planes.

\section{ACKNOWLEDGMENTS}

It is a pleasure to acknowledge important conversations with Bob Markiewicz. This work is supported by the US Department of Energy contract W-31-109-ENG-38, and benefited from the allocation of supercomputer time at the NERSC and the Northeastern University Advanced Scientific Computation Center (ASCC) and Institute of Advanced Computing (IAC), Tampere. One of us (S.S.) acknowledges Suomen Akatemia, Vilho, Yrjö ja Kalle 
Väisälän Rahasto, Tekniikan Edistämissäätiö and Jenny ja Antti Wihurin Rahasto for financial support. 


\section{REFERENCES}

[1] A. Bansil and M. Lindroos, Phys. Rev. Lett. 83, 5154(1999).

[2] A. Bansil and M. Lindroos, J. Phys. Chem. Solids 59, 1879(1998).

[3] M. C. Asensio, J. Avila, L. Roca, A. Tejeda, G.D. Gu, M. Lindroos, R.S. Markiewicz, and A. Bansil, to be published.

[4] P.V. Bogdanov, et al., Phys. Rev. Lett. (2001), cond-mat/0005394.

[5] D.L. Feng, et al., Phys. Rev. Lett. 86, 5550(2001).

[6] Y.D. Chuang, et al., Phys. Rev. Lett. 83, 3717(1999); Y.D. Chuang, et al., condmat/0107002.

[7] See e.g., "Spectroscopies in Novel Superconductors", edited by A. Bansil, R. Markiewicz, S. Sridhar and D. Liebenberg [J. Phys. Chem. Solids 59, No 10-12 (1998)], and other volumes in this series for discussions of these and related issues.

[8] J.B. Pendry, Surface Sci. 57, 679(1976); J.F.L. Hopkinson, J.B. Pendry and D.J. Titterington, Computer Phys. Commun. 19, 69(1981).

[9] A. Bansil and M. Lindroos, J. Phys. Chem. Solids 56, 1855(1995).

[10] M. Lindroos and A. Bansil, Phys. Rev. Lett. 75, 1182(1995).

[11] M. Lindroos and A. Bansil, Phys. Rev. Lett. 77, 2985(1996).

[12] C.G. Larsson, Surface Science 152/153, 213(1985).

[13] M. Cardona and L. Ley, Photoemission in Solids I: General Principles, Spinger-Verlag Berlin 1978.

[14] C. Kunz in Photoemission in Solids II, edited by L. Ley and M. Cardona, SpringerVerlag Berlin 1979, p313.

[15] J.E. Inglesfield and E.W. Plummer on S.D. Kevan (ed.), Angle Resolved Photoemission, Theory and Current Applications, Elsevier 1992.

[16] M. Lindroos, Physica Scripta 25, 788(1982).

[17] S. Sahrakorpi, M. Lindroos and A. Bansil, submitted to Phys. Rev. B.

[18] A. Bansil et al., J. Phys. Chem. Solids 54, 1185(1993); M. Lindroos et al., Physica C212, 347(1993).

[19] K. Gofron, J. C. Campuzano, A. A. Abrikosov, M. Lindroos, A. Bansil, H. Ding, D. Koelling and B. Dabrowski, Phys. Rev. Lett. 73, 3302(1994).

[20] Angle-resolved Photoemission, Theory and Current Applications edited by S.D. Kevan, Elsevier, 1992.

[21] G.D. Mahan, Phys. Rev. B2, 4334(1970).

[22] C. Caroli, D. Lederer-Rozenblatt, B. Roulet, D. Saint-James, Phys. Rev. B8, 4552(1973).

[23] P.J. Durham, B.L. Gyorffy and A.J. Pindor, J. Phys. F: Metal Phys. 10, 661(1980).

[24] J. Braun, G. Thörner and G. Borstel, Phys. Stat. Sol. B130, 643(1985).

[25] M. Grass, J. Braun and G. Borstel, Phys. Rev. B50, 14827(1994).

[26] H.M. Fretwell, et al., Phys. Rev. Lett. 84, 4449(2000).

[27] S.V. Borisenko, et al., Phys. Rev. Lett. 84, 4453(2000); A.A. Kordyuk, et al., condmat/0104294.

[28] T. Sato, et al., Phys. Rev. B63, 132502(2001).

[29] P. Aebi, et al., Phys. Rev. Lett. 72, 2757(1994).

[30] C.F. Behrens, et al., Phys. Rev. Lett. 76, 1553(1996).

[31] N. Saini, et al., Phys. Rev. Lett. 79, 3467(1997). 
[32] X.J. Zhou, et al., Science 286, 268(1999).

[33] T. Valla, et al., Phys. Rev. Lett. 85, 828(2000).

[34] A. Zakharov et al. , Phys. Rev. B 61, 115(2000).

[35] S. Rast et al., Europhys. Lett. 51, 103(2000).

[36] Wave length of light is $\sim 600 \AA$ while the unit cell dimensions are $\sim 30 \AA$.

[37] Seppo Sahrakorpi, Ph.D. Thesis, Tampere University of Technology (2001), to be published.

[38] B. Segall, Phys. Rev. 105, 108(1957).

[39] P. E. Mijnarends and A. Bansil, J. Phys.:Condens. Matter 2, 911(1990).

[40] It is straightforward to mimic correlation effects in the present framework by introducing a self-energy correction to the Hamiltonian. Further work is however needed in this regard, especially for incorporating strong correlations effects in the ARPES simulations.

[41] $\Sigma_{i}^{\prime \prime}$ and $\Sigma_{f}^{\prime \prime}$ are the imaginary parts of the initial and final state self-energies, respectively, used to include lifetime effects. For a damped propagator the full-width-at-halfmaximum (FWHM) of the peak is roughly given by $2 \Sigma^{\prime \prime}$. See, N.V. Smith, P.Thiry and Y. Petroff, Phys. Rev. B 47, 15476(1993).

[42] V.N. Strocov, H.I. Starnberg and P.O. Nilsson, J. Phys.: Condens. Matter 8, 7539 (1996).

[43] R. Courths, S. Löbus, S. Halilov, T. Scheunemann, H. Gollisch and R. Feder, Phys. Rev. B 60, 8055 (1999).

[44] M. Lindroos and A. Bansil, J. Phys. Chem. Solids 52, 1447(1991).

[45] When the states are broadened, the effect of these gaps will of course be reduced.

[46] While these weights are relevant for analyzing the behavior of the momentum matrix element, one should keep in mind that these do not include the effect of the radial part of the wavefunction as seen from Eqs. 10 and 11. 


\section{TABLES}

TABLE I. Magnitude and phase of the matrix element $M_{x}^{\gamma, \delta}$ is given for various sites $\gamma$ and $\delta$ for transition 1 in Fig. 7 (or Fig. 8) for both the antibonding initial state $a$ and the bonding initial state $b$ at M. Note that Figs. 7 and 8 only refer to state $a$, but that this table also gives results for the state $b$.

\begin{tabular}{l|r|r|r|r|r|r|}
\hline \hline element & $\gamma$ & $\delta$ & \multicolumn{2}{|c|}{ Antibonding state $a$} & \multicolumn{2}{|c|}{ Bonding state $b$} \\
\hline & & & $\left|M_{x}^{\gamma, \delta}\right|$ & phase & $M_{x}^{\gamma, \delta}$ & phase \\
\hline $\mathrm{Ca}$ & 1 & 1 & 0.0279 & 35.6 & 0.0000 & - \\
$\mathrm{Cu}$ & 2 & 1 & 0.0209 & 35.6 & 0.0191 & 16.8 \\
$\mathrm{Cu}$ & 2 & 2 & 0.0209 & 35.6 & 0.0191 & -163.2 \\
$\mathrm{O}_{C u, x}$ & 3 & 1 & 0.4309 & 35.6 & 0.3111 & 16.8 \\
$\mathrm{O}_{C u, x}$ & 3 & 2 & 0.4309 & 35.6 & 0.3111 & -163.2 \\
$\mathrm{O}_{C u, y}$ & 4 & 1 & 0.0518 & 35.6 & 0.0784 & 16.8 \\
$\mathrm{O}_{C u, y}$ & 4 & 2 & 0.0518 & 35.6 & 0.0784 & -163.2 \\
$\mathrm{Sr}$ & 5 & 1 & 0.0158 & 35.6 & 0.0081 & 16.8 \\
$\mathrm{Sr}$ & 5 & 2 & 0.0158 & 35.6 & 0.0081 & -163.2 \\
$\mathrm{O}_{S r}$ & 6 & 1 & 0.0316 & 35.6 & 0.0140 & 16.8 \\
$\mathrm{O}_{S r}$ & 6 & 2 & 0.0316 & 35.6 & 0.0140 & -163.2 \\
$\mathrm{Bi}$ & 7 & 1 & 0.0314 & -144.4 & 0.0366 & -163.2 \\
$\mathrm{Bi}$ & 7 & 2 & 0.0314 & -144.4 & 0.0366 & 16.8 \\
$\mathrm{O}_{B i}$ & 8 & 1 & 0.0167 & -144.4 & 0.0417 & -163.2 \\
$\mathrm{O}_{B i}$ & 8 & 2 & 0.0167 & -144.4 & 0.0417 & 16.8 \\
\hline \hline
\end{tabular}

TABLE II. Wavefunction of the antibonding state $a$ at M-point is characterized in terms of weights (in percent) defined as $\sum_{\delta}\left|C_{L}^{\gamma \delta}\right|^{2}$ associated with different sites $\gamma$ (see Eq. 10). Not all angular momentum channels are listed. Channels with small weights are omitted for brevity; the total weight for this reason adds to less than 100 percent. 46.

\begin{tabular}{|c|c|c|c|c|c|c|}
\hline$\gamma$ & Tot. & $s$ & $p_{z}$ & $p_{x}$ & $d_{3 z^{2}-r^{2}}$ & $d_{x^{2}-y^{2}}$ \\
\hline $\mathrm{Ca}$ & & & & & & \\
\hline $\mathrm{Bi}$ & 12 & & 7 & 5 & & \\
\hline $\mathrm{Sr}$ & & & & & & \\
\hline $\mathrm{Cu}$ & 43 & 3 & & & 12 & 28 \\
\hline $\mathrm{O}_{C u, x}$ & 24 & & & 24 & & \\
\hline $\mathrm{O}_{C u, y}$ & & & & & & \\
\hline $\mathrm{O}_{B i}$ & 4 & & 4 & & & \\
\hline $\mathrm{O}_{S r}$ & 6 & & 6 & & & \\
\hline
\end{tabular}


TABLE III. Same as the caption to Table II, except that this table refers to the bonding state $b$ at the M-point.

\begin{tabular}{|c|c|c|c|c|c|c|}
\hline$\gamma$ & Tot. & $s$ & $p_{z}$ & $p_{x}$ & $d_{3 z^{2}-r^{2}}$ & $d_{x^{2}-y^{2}}$ \\
\hline $\mathrm{Ca}$ & & & & & & \\
\hline $\mathrm{Bi}$ & 10 & 4 & 6 & & & \\
\hline $\mathrm{Sr}$ & & & & & & \\
\hline $\mathrm{Cu}$ & 29 & 2 & & & 7 & 20 \\
\hline $\mathrm{O}_{C u, x}$ & 13 & & & 13 & & \\
\hline $\mathrm{O}_{C u, y}$ & 4 & & 4 & & & \\
\hline $\mathrm{O}_{B i}$ & 24 & & 6 & 18 & & \\
\hline $\mathrm{O}_{S r}$ & 11 & & 11 & & & \\
\hline
\end{tabular}

TABLE IV. Same as the caption to Table II, except that this table refers to the final state of transition 1 in Fig. 7 (or Fig. 8) involving the excitation of the antibonding initial state $a$.

\begin{tabular}{|c|c|c|c|c|c|c|c|}
\hline$\gamma$ & Tot. & $s$ & $p_{z}$ & $p_{x}$ & $d_{x z}$ & $d_{x^{2}-y^{2}}$ & $f_{z^{3}}$ \\
\hline $\begin{array}{l}\mathrm{Ca} \\
\mathrm{Bi}\end{array}$ & 7 & \multirow{7}{*}{10} & \multirow{7}{*}{3} & \multirow{7}{*}{29} & \multirow{7}{*}{2} & \multirow{7}{*}{6} & \multirow[t]{7}{*}{5} \\
\hline $\mathrm{Sr}$ & 18 & & & & & & \\
\hline $\mathrm{Cu}$ & 29 & & & & & & \\
\hline $\mathrm{O}_{C u, x}$ & 18 & & & & & & \\
\hline $\mathrm{O}_{C u, y}$ & 4 & & & & & & \\
\hline $\mathrm{O}_{B i}$ & & & & & & & \\
\hline $\mathrm{O}_{S r}$ & & & & & & & \\
\hline
\end{tabular}


TABLE V. Components $\sum_{\delta} M_{x, L, L^{\prime}}^{\gamma \delta}$ of the momentum matrix element (see Eqs. 14-17) for transition 1 marked in Fig. 7 (or Fig. 8) which involves excitation of the antibonding initial state $a$ at M-point. Only sites and channels with significant weight are listed.

\begin{tabular}{|c|c|c|c|}
\hline$\gamma$ & Channel: $L \rightarrow L^{\prime}$ & $M_{x, L, L^{\prime}}^{\gamma}$ & $\sum_{L L^{\prime}} M_{x, L, L^{\prime}}^{\gamma}$ \\
\hline $\mathrm{O}_{C u, x}$ & $\begin{array}{l}p_{x} \rightarrow s \\
p_{x} \rightarrow d_{3 z^{2}-r^{2}} \\
p_{x} \rightarrow d_{x^{2}-y^{2}}\end{array}$ & $\begin{array}{l}+0.12 \\
+0.04 \\
+0.27 \\
\end{array}$ & +0.43 \\
\hline $\mathrm{Cu}$ & $\begin{array}{l}s \rightarrow p_{x} \\
d_{x^{2}-y^{2}} \rightarrow p_{x} \\
d_{x^{2}-y^{2}} \rightarrow f_{x z^{2}} \\
d_{x^{2}-y^{2}} \rightarrow f_{x\left(x^{2}-3 y^{2}\right)} \\
d_{3 z^{2}-r^{2}} \rightarrow p_{x} \\
d_{3 z^{2}-r^{2}} \rightarrow f_{x z^{2}}\end{array}$ & $\begin{array}{r}+0.01 \\
+0.07 \\
-0.02 \\
-0.03 \\
+0.03 \\
-0.04\end{array}$ & +0.02 \\
\hline
\end{tabular}




\section{FIGURES}

FIG. 1. Body-centered tetragonal crystal structure of Bi2212. (a) and (b) give (110) and (100) cross-sections through the structure, respectively. (c) depicts the arrangement of atoms in different layers. Various basis atoms are identified by the pairs of indices $(\gamma, \delta)$ discussed in the text.

FIG. 2. Band structure of Bi2212 in the vicinity of the Fermi energy $E_{F}$ (shown by the horizontal dashed line) along the symmetry lines $\Gamma-M-X-\Gamma$. Note that the $B i$ - $O$ pockets around $\mathrm{M}$ in the standard LDA band structure have been lifted above the $E_{F}$; see text for details of crystal potential. $a$ and $b$ are the antibonding and bonding states at M, respectively, arising from the $\mathrm{CuO}_{2}$ planes. $\mathrm{c}$ and $c^{\prime}$ are $\mathrm{Bi}-\mathrm{O}$ related states.

FIG. 3. ARPES intensity for emission from the $\mathrm{CuO}_{2}$ plane related antibonding and bonding states at $\bar{M}$ as a function of the photon energy.

FIG. 4. ARPES intensity from the antibonding state $a$ at $\bar{M}$ as a function of the photon energy for four different values of the width of the final state determined by the parameter $\Sigma_{f}^{\prime \prime}$ 41].

FIG. 5. Polarization dependence of the ARPES spectra at $\bar{M}$ for $22 \mathrm{eV}$ light. Energy distribution curve (EDC) including realistic initial state broadening for light polarized along the $x$-axis is shown (thick line); thin line gives the EDC computed by using an artificially small damping of initial states to reveal the spectral peaks $a, b, c$ and $c^{\prime}$ clearly. EDC's have not been convoluted with the Fermi function. Inserts in the shapes of number eight on top of various peaks are polar plots of peak intensity as the direction of the polarization vector $(\phi)$ is varied and possess the $\cos ^{2} \phi$ dependence in accord with Eq. 20. $\phi$ is measured with respect to the $x$-axis or the $C u-O_{x}$ bond direction; as seen in the inset in the top part of the figure, the detector (D) is assumed to lie in the vertical plane through the $x$-axis.

FIG. 6. Computed one-step ARPES intensity in BISCO at $\bar{M}$ from the antibonding state $a$ as a function of the photon energy is compared with the corresponding result based on the momentum matrix element $\left|M_{x}\right|^{2}$ for bulk transitions (see Eq. 14). A small final state damping is used to reveal features clearly. The relevant final state bands are shown as a function of $k_{\perp}$ and energy in the lower figure where the energy scale has been shifted to correspond to photon energy. 
FIG. 7. Total matrix element $\left|M_{x}\right|$ (topmost panel) for various transitions from the antibonding state $a$ over the transition energy range of $10-27 \mathrm{eV}$ is decomposed into contributions from various "pairs" of atomic sites $\gamma$ (panels (b)-(e)) as described in Eqs. 14 16. Down arrows indicate a negative value of $M_{x}^{\gamma}$. Sites not shown yield a negligible contribution. Transitions labelled 1-7 (connected by dashed lines for ease of identification across the panels) are discussed in the text to highlight various interference effects.

FIG. 8. Same as the caption to Figure 7 with the two top panels (a) and (b) reproduced from Figure 7 for ease of reference. Panels (c)-(e) give the decomposition of $O_{C u, x}$ results in (b) into various angular momentum channels $\sum_{\delta} M_{x, L, L^{\prime}}^{\gamma \delta}$ (see Eq. 16).

FIG. 9. $\quad k_{\perp}$ dependence of the total momentum matrix element $\left|M_{x}\right|$ for a few selected transitions from the antibonding initial state $a$ at $\bar{M}$. Transitions 1-4 are the same as those in Figs. 7 and 8. Transition $2 \mathrm{~b}$ (or $3 \mathrm{~b}$ ) possesses energy close to 2 (or 3 ) but its intensity is zero (or quite small) for $k_{\perp}=0$, or equivalently, at $\bar{M}(\pi, 0,0)$. 


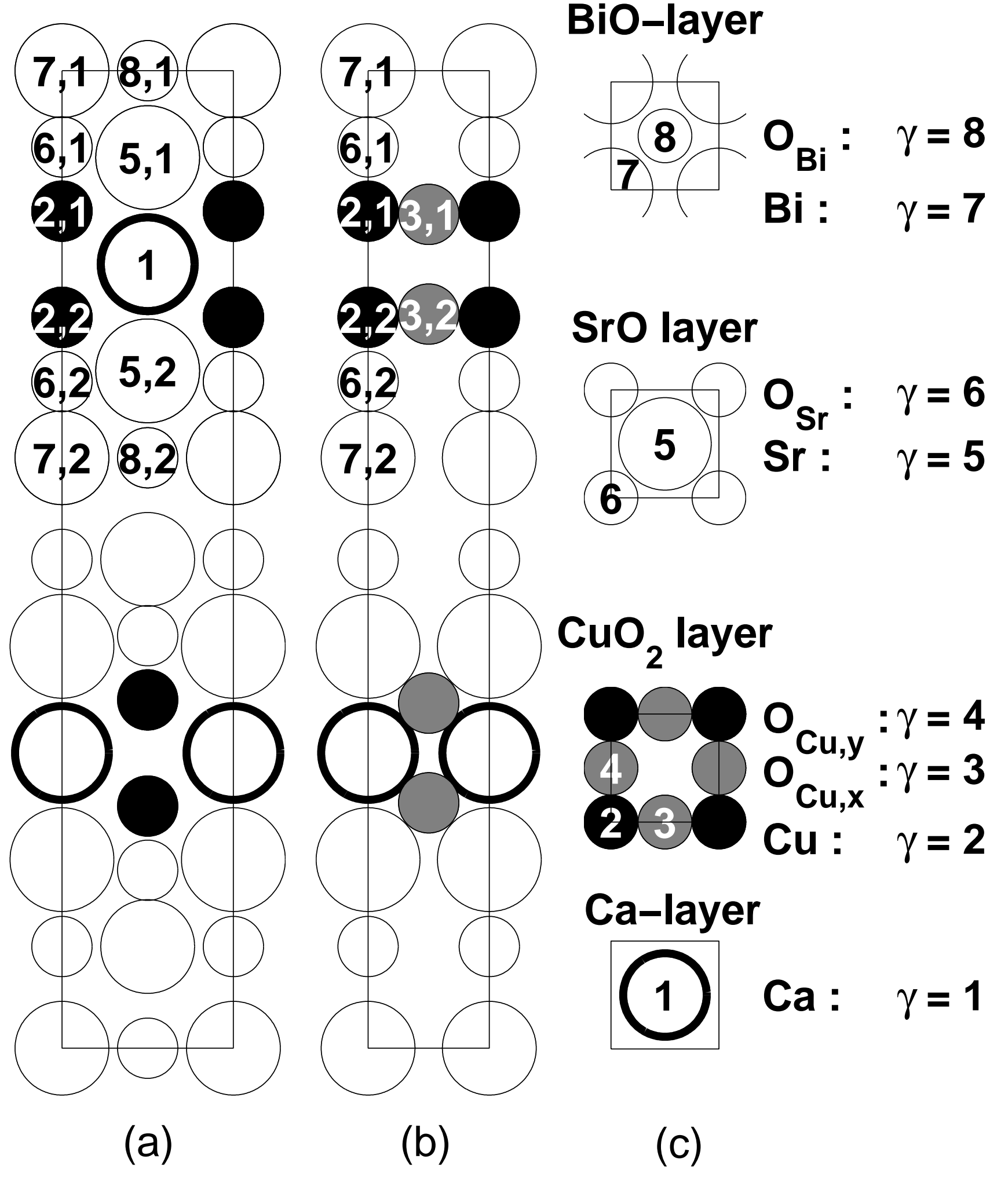




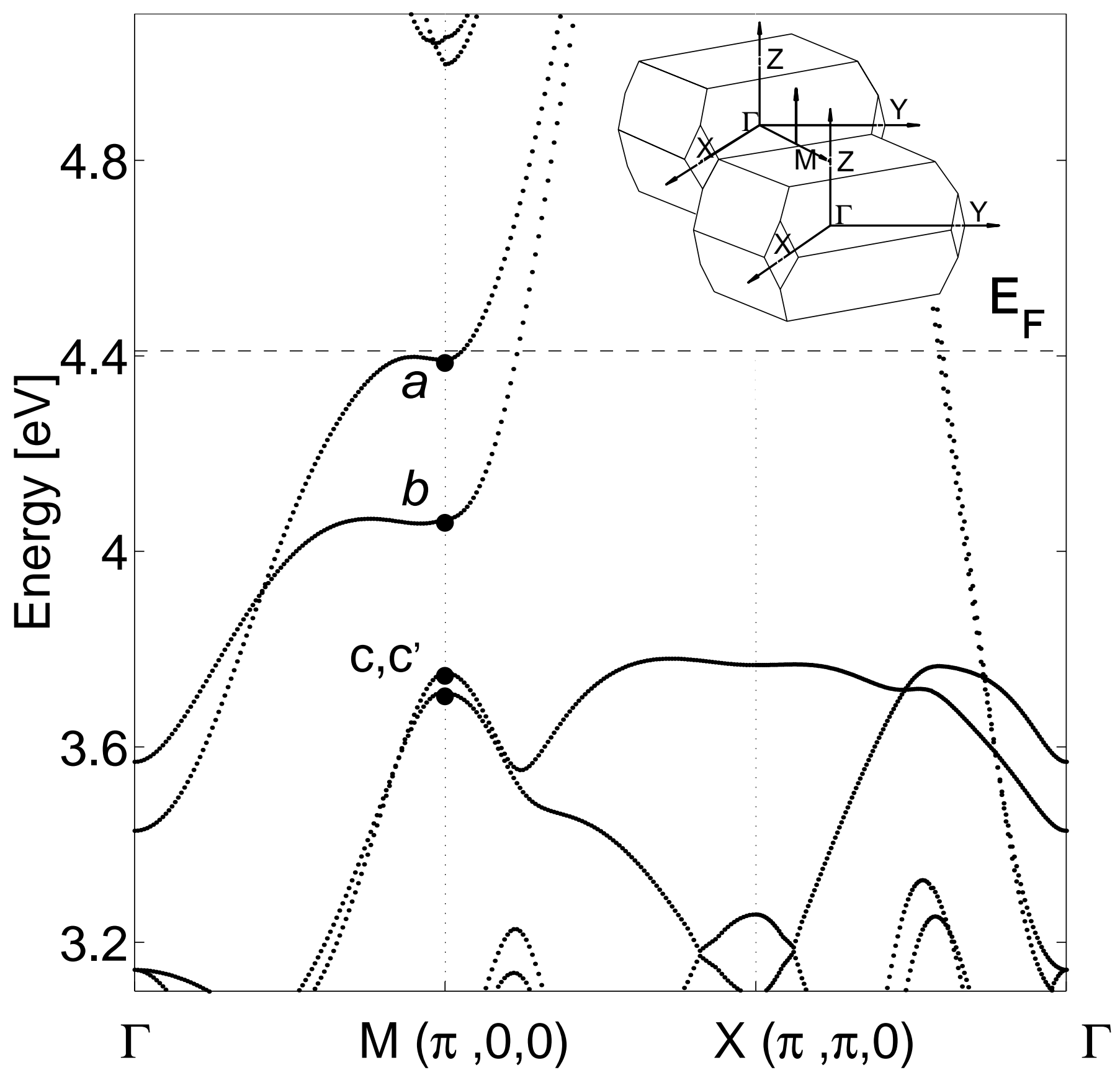




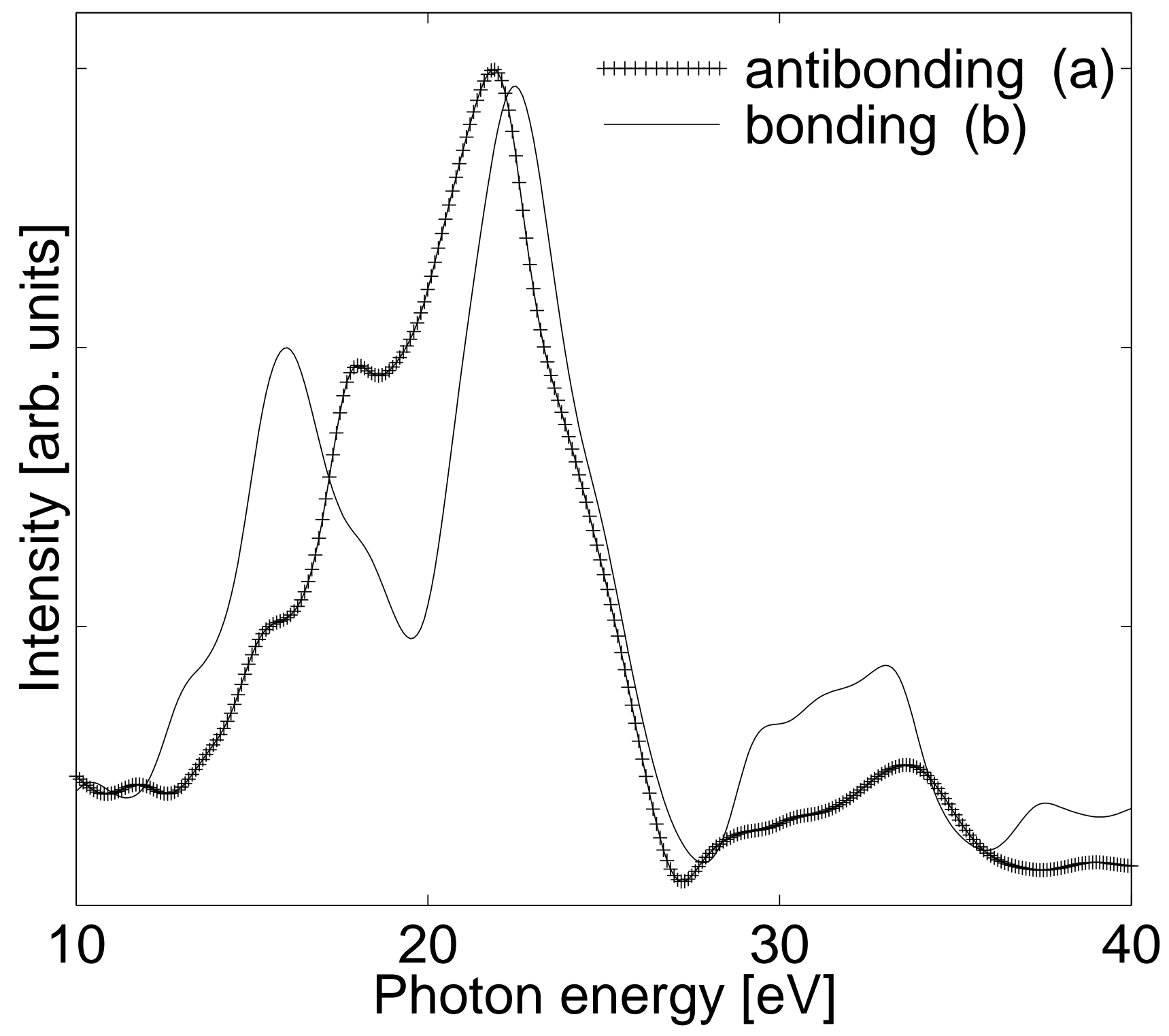



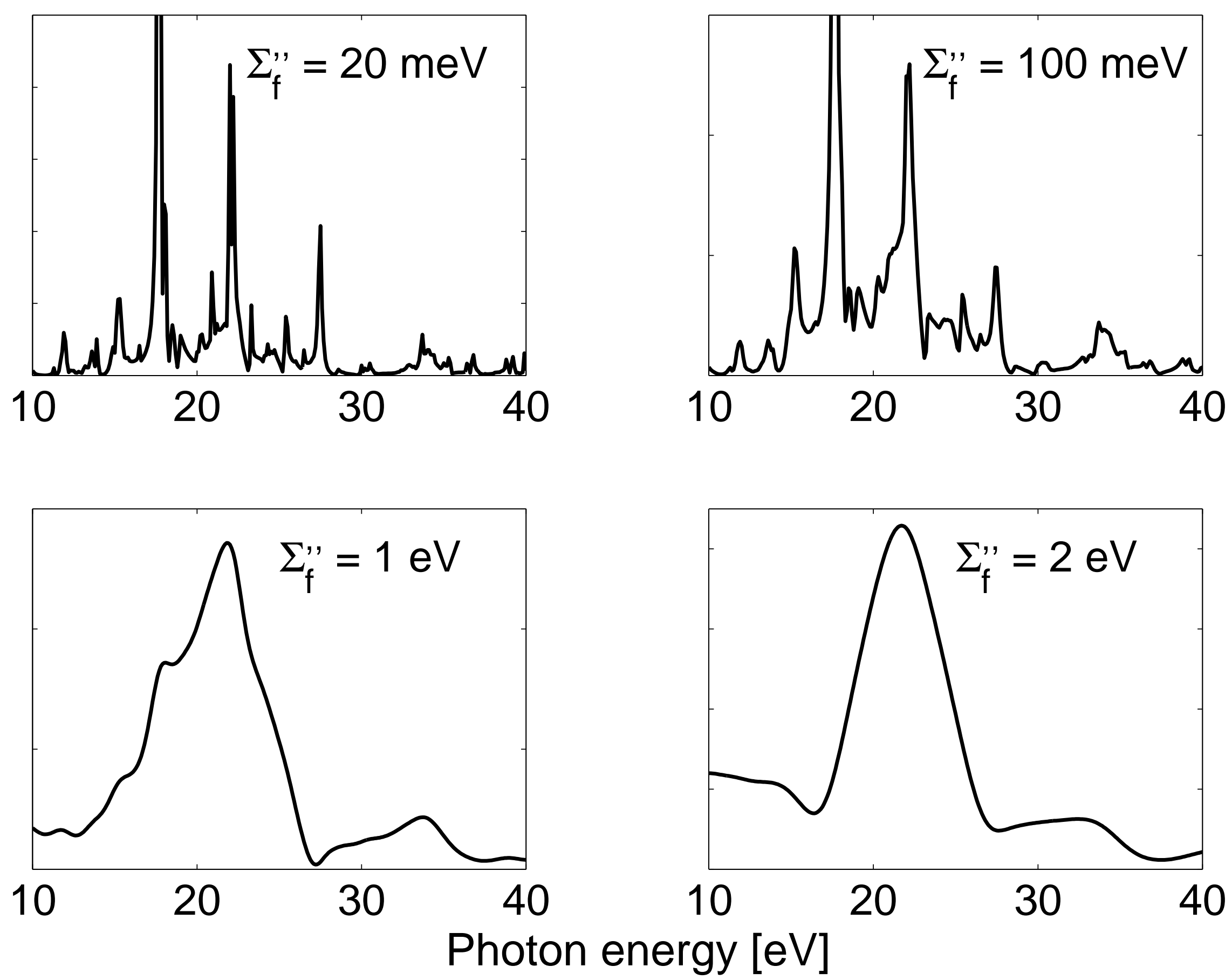


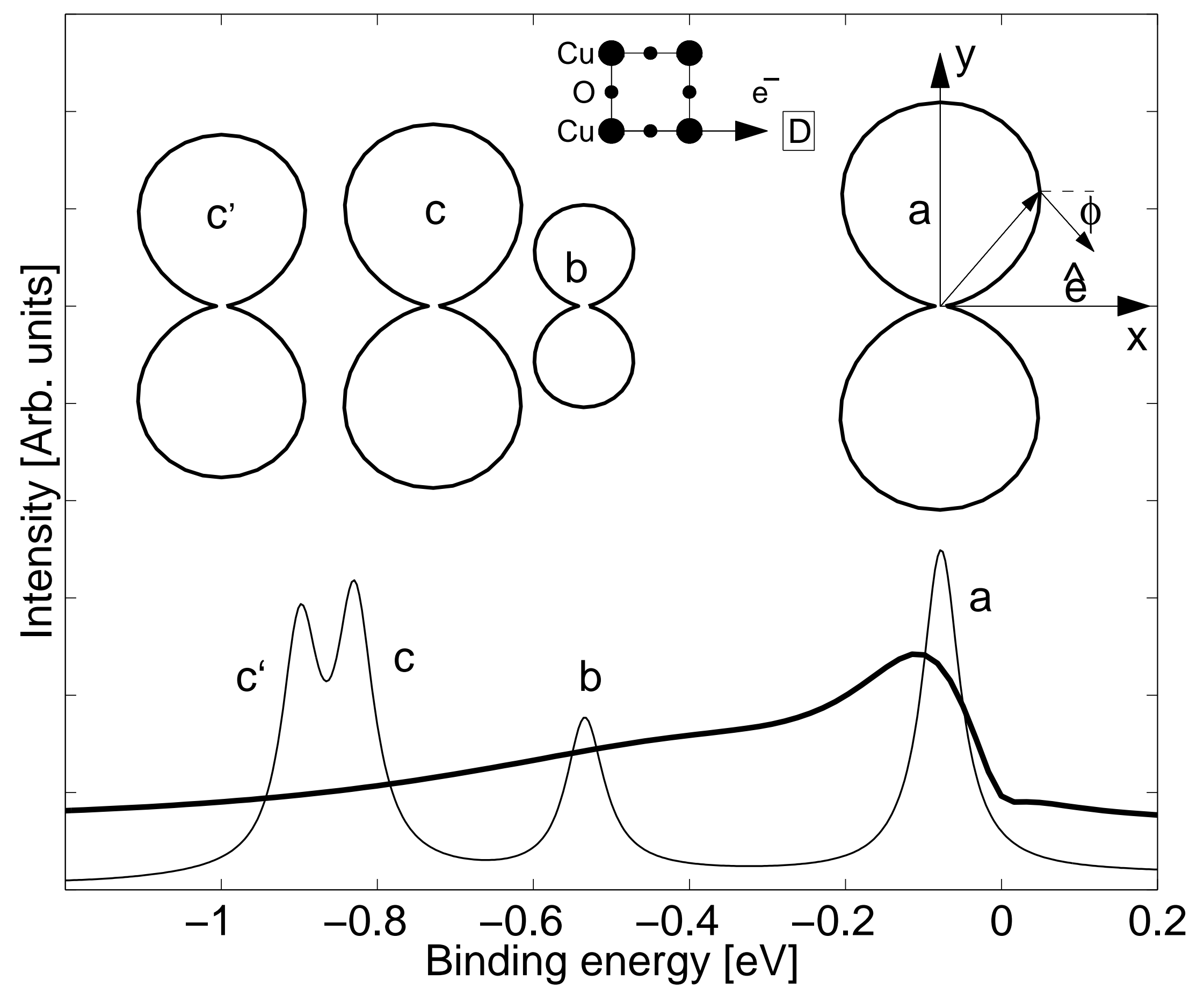



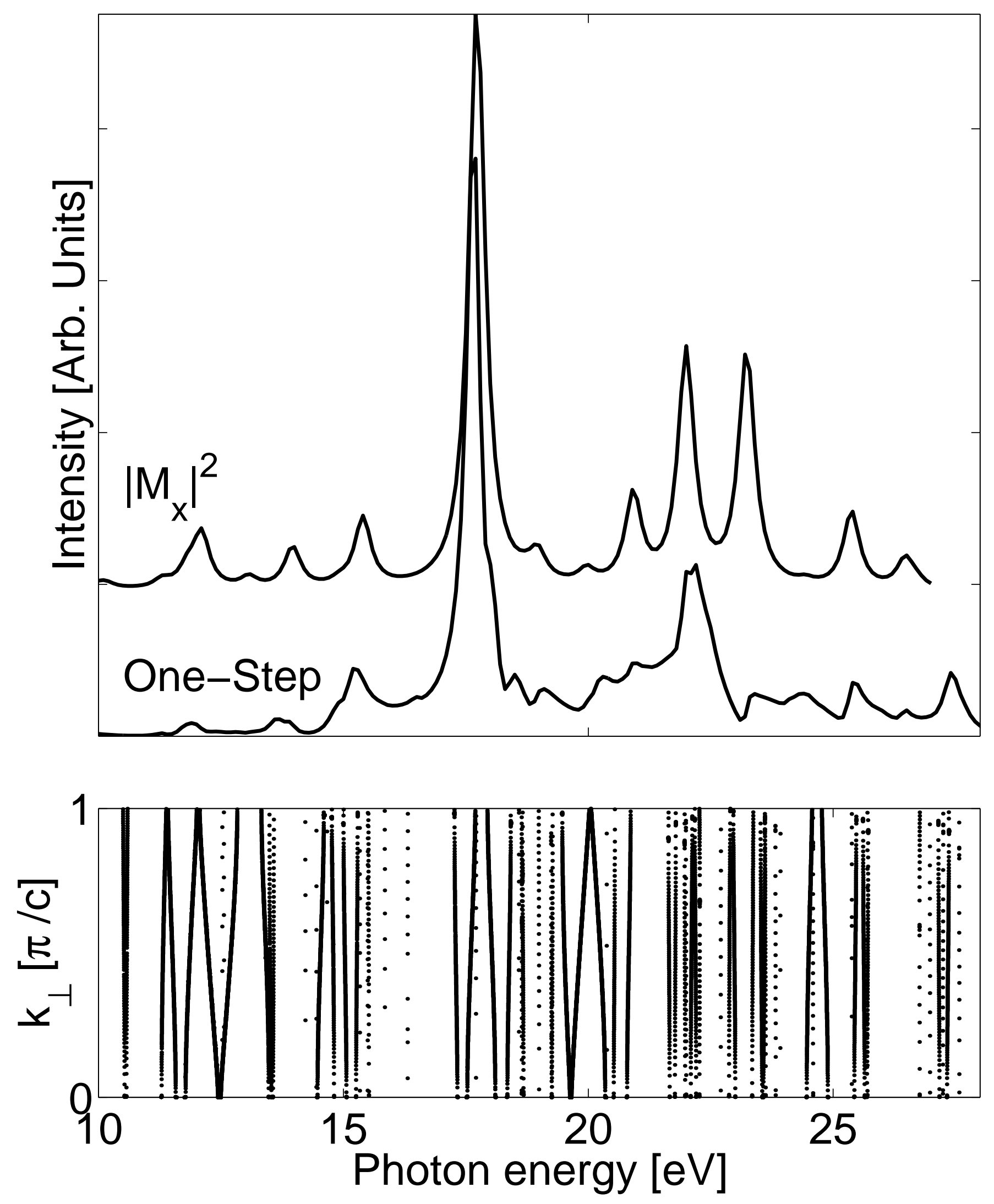


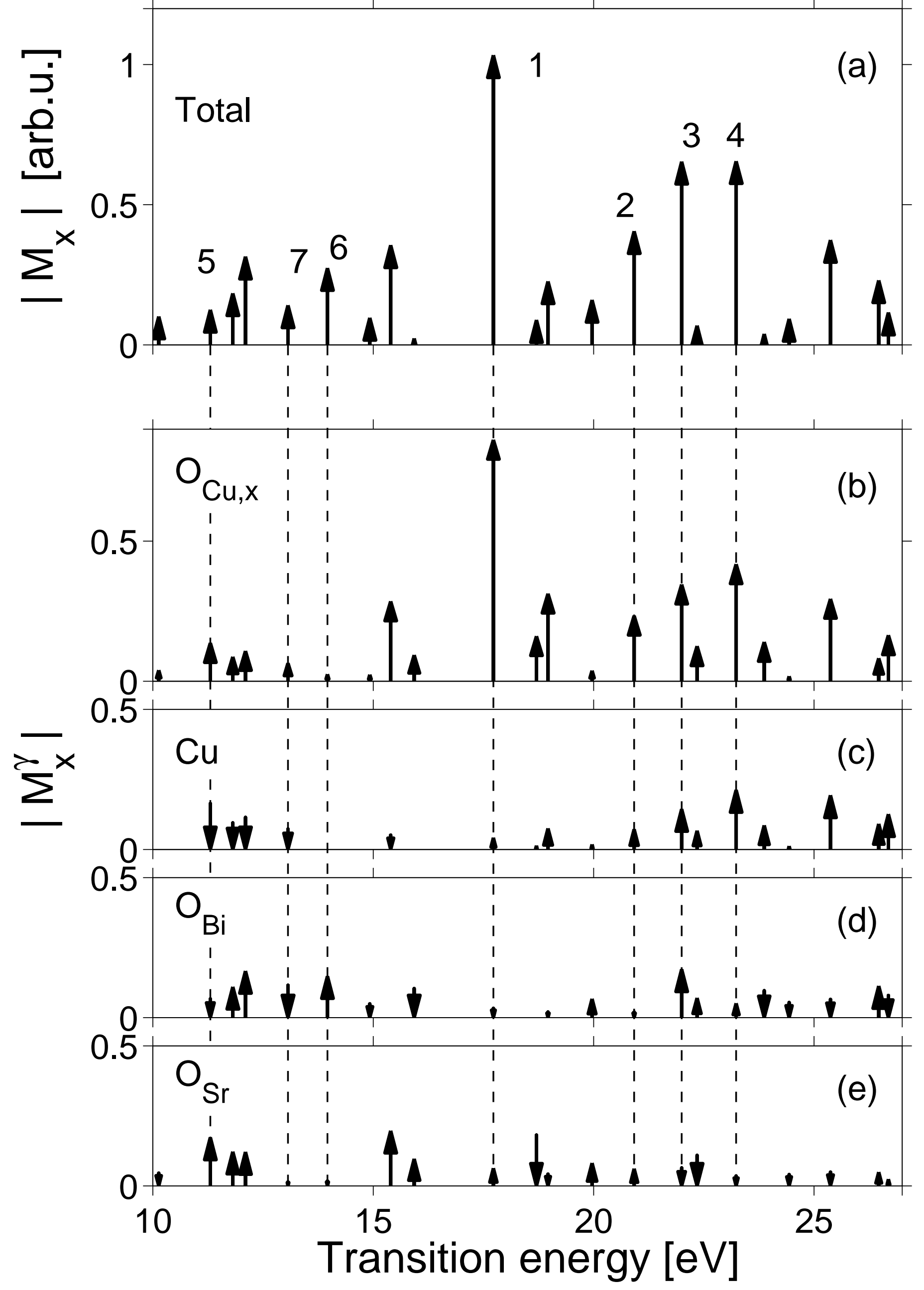




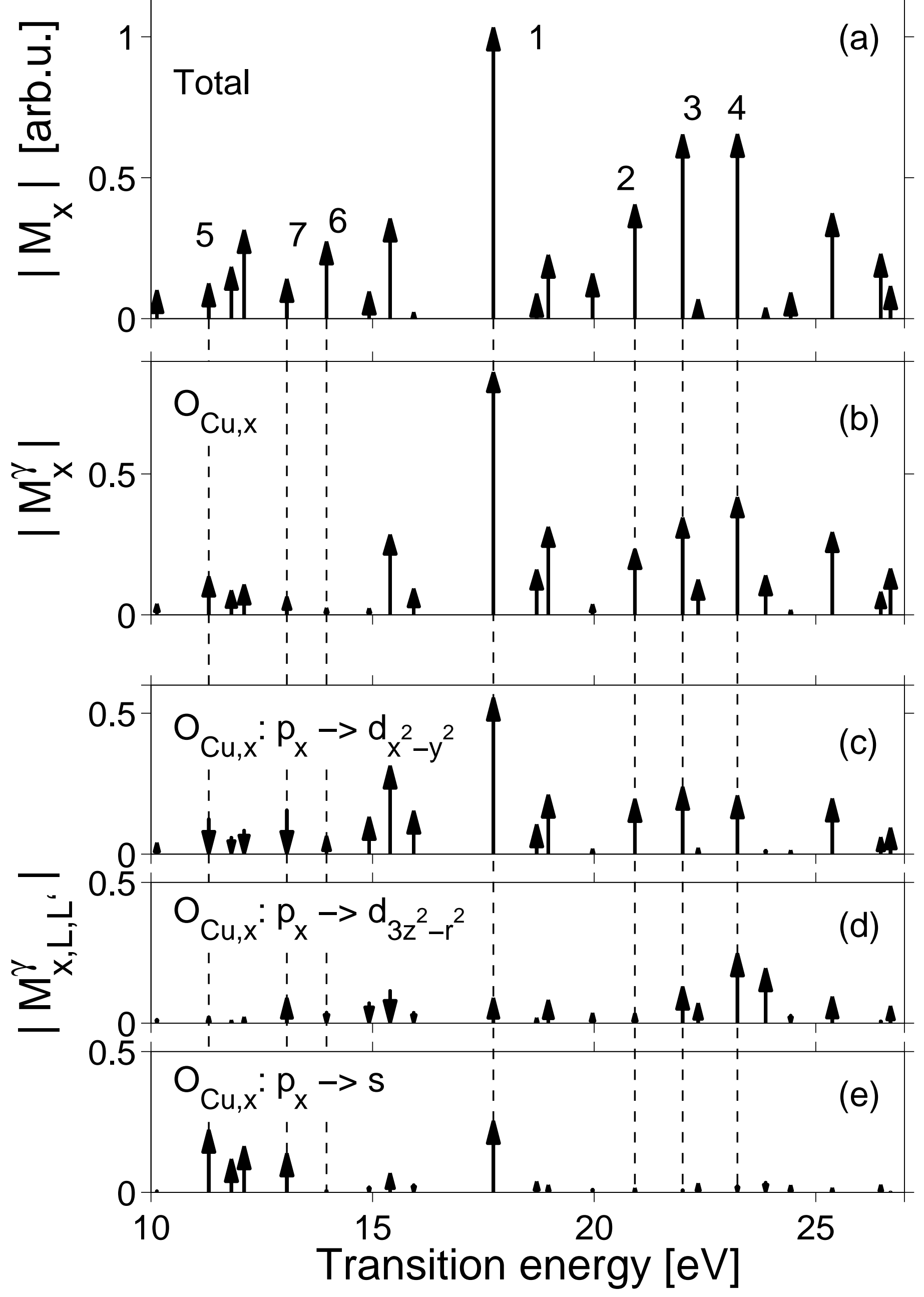




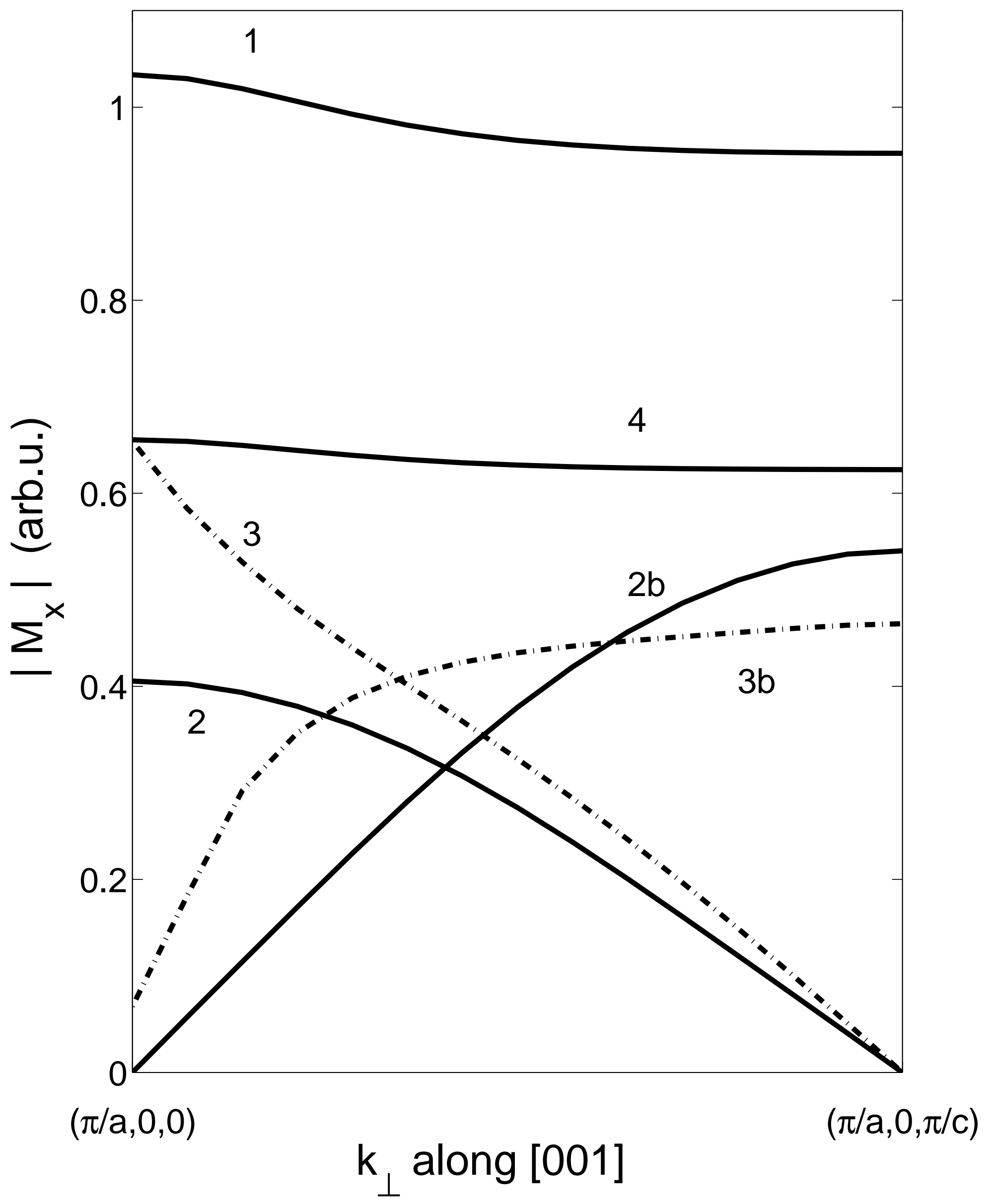

